\title{
An Update on the Female Presence at the IOI
}

\author{
Stefano MAGGIOLO \\ London, U.K. \\ e-mail:s.maggiolo@gmail.com
}

\begin{abstract}
The gender ratio in computer science is already very unbalanced; we gathered statistics about the gender of contestants and leaders to show that the female participation at the IOI is even lower. We look at existing programs trying to increase gender balance at a national level and offer some suggestions, including (re)-introducing mixed gender requirements for complete teams.
\end{abstract}

Keywords: gender imbalance.

\section{Introduction}

There is no need for statistics to realize how much the IOI are unbalanced in terms of gender distribution: it is far too easy to notice the gender skew just by walking around during the IOI week, or by looking at the audience of the opening ceremony.

But we need statistics when we want to compare the IOI with the general trend in computer science and in math-intensive fields, and if we want to track progress. For example, the organization of the IMO is now recording the gender distribution of the contestants for recent editions, and many past editions have also been back-filled (International Mathematical Olympiad).

We asked the national delegations to provide this piece of historical data, and to share the programs they have in place to improve gender balance. This article is mainly about presenting the results of this survey (section 2), and comparing the IOI's gender ratio with those of other relevant institutions and companies (section 3).

At the international level there have not been many initiatives in this direction. The main one happened in 1995, when the Netherlands, as the organizing country of the IOI, allowed delegations of five contestants (instead of the usual four) when the team was gender mixed. Despite having a reasonable success, with four times as many female as the average edition, the program was not reproposed by other hosting countries, nor picked up by the International Committee.

In section 4 we present arguments for the IOI to adopt more actions to reach female contestants, in accordance with its goal of promoting computer science among young 
people. Finally, in section 5, we offer suggestions for possible initiatives, both at the national and international level.

\section{Data}

We prepared a questionnaire to collect historical data regarding female participation at IOI, and we sent it to the contact person of each member country, as listed in the IOI's website. Given the low number of replies, the questionnaire was also extended to the two mailing lists ioi-announce and ioi-discuss.

\subsection{Format}

The questionnaire was composed of three parts, asking:

1. To describe the national programs having effects on the female participation at the IOI.

2. To estimate the total and female participation at three stages of the team selection: the "base" (all the participants), the "national olympiad", and the "training".

3. To mark the gender of each member of the official team (contestants and leaders) participating in the past IOI editions.

Names and pictures (where present) of the team members were obtained from the IOI statistics website (Kalinicenko). The same source was used to get all the participation numbers we present in the rest of the section.

\subsection{Participation}

Overall, 36 delegations responded (44\% of the 81 countries participating in IOI 2014). We completed the gender assignment for the remaining 61 countries, for what we could infer from several, not necessarily correct, sources: gender-defining names, pictures in IOI-related websites, notes in the IOI newsletters, and generic web search. These approaches still left about $5 \%$ to $10 \%$ of the contestants marked as "not sure" for years up to 2001 , and $0 \%$ to $5 \%$ since 2002 , especially for countries that lack gender-defining names.

Given the generally low female participation, false negatives could change significantly the numerical results, even if a big change is not very likely. In any case, we believe that our conclusions are not impacted, especially given that the number of contestants of the last few editions for which we lack the assignment is very low. We encourage the IOI to collect the gender of the participants and make it available on the statistics website (Kalinicenko), to ease future investigations. 


\subsection{Results}

Per year results. Table 1 contains the gender data aggregated by year, and Fig. 1 shows the percentage ${ }^{1}$ of female participants, as contestants and leaders separately.

It is clear from the figure that there is not an obvious trend in the female participation. For leaders, if any, there is a slightly decreasing trend, but the data for years before 2000 is far from complete. For contestants, the period between years 1998 and 2006 is quite striking, having $2 \%$ or less female contestants for 9 consecutive years.

Table 1

Per-year participation at IOI, broke down by gender and role (leader or contestant). The columns "F" and " $\mathrm{M}$ " indicate the number of female and male participants, respectively; the column "?" the number of participants for which we were unable to assign a gender; the column "F \%" the percentage of female participants among those we were able to assign a gender to.

\begin{tabular}{|c|c|c|c|c|c|c|c|c|c|c|}
\hline \multirow[t]{2}{*}{ Year } & \multicolumn{5}{|c|}{ Leaders } & \multicolumn{5}{|c|}{ Contestants } \\
\hline & Tot & $\mathrm{F}$ & $\mathrm{M}$ & $?$ & $\mathrm{~F} \%$ & Tot & $\mathrm{F}$ & $\mathrm{M}$ & $?$ & $\mathrm{~F} \%$ \\
\hline 1989 & 14 & 0 & 6 & 8 & 0.0 & 37 & 1 & 21 & 15 & 4.5 \\
\hline 1990 & 2 & 1 & 1 & 0 & 50.0 & 38 & 2 & 32 & 4 & 5.9 \\
\hline 1991 & 17 & 2 & 15 & 0 & 11.8 & 68 & 1 & 63 & 4 & 1.6 \\
\hline 1992 & 79 & 10 & 65 & 4 & 13.3 & 171 & 7 & 151 & 13 & 4.4 \\
\hline 1993 & 29 & 2 & 27 & 0 & 6.9 & 107 & 5 & 94 & 8 & 5.1 \\
\hline 1994 & 88 & 7 & 78 & 3 & 8.2 & 189 & 8 & 165 & 16 & 4.6 \\
\hline 1995 & 30 & 2 & 28 & 0 & 6.7 & 210 & 19 & 172 & 19 & 9.9 \\
\hline 1996 & 106 & 8 & 95 & 3 & 7.8 & 218 & 3 & 200 & 15 & 1.5 \\
\hline 1997 & 95 & 10 & 81 & 4 & 11.0 & 221 & 7 & 199 & 15 & 3.4 \\
\hline 1998 & 51 & 5 & 46 & 0 & 9.8 & 241 & 2 & 224 & 15 & 0.9 \\
\hline 1999 & 54 & 6 & 48 & 0 & 11.1 & 172 & 2 & 158 & 12 & 1.3 \\
\hline 2000 & 137 & 18 & 116 & 3 & 13.4 & 268 & 4 & 251 & 13 & 1.6 \\
\hline 2001 & 75 & 8 & 67 & 0 & 10.7 & 261 & 5 & 241 & 15 & 2.0 \\
\hline 2002 & 55 & 5 & 50 & 0 & 9.1 & 272 & 4 & 256 & 12 & 1.5 \\
\hline 2003 & 50 & 5 & 45 & 0 & 10.0 & 261 & 3 & 247 & 11 & 1.2 \\
\hline 2004 & 148 & 12 & 134 & 2 & 8.2 & 298 & 4 & 286 & 8 & 1.4 \\
\hline 2005 & 138 & 10 & 127 & 1 & 7.3 & 281 & 5 & 274 & 2 & 1.8 \\
\hline 2006 & 142 & 10 & 130 & 2 & 7.1 & 289 & 5 & 273 & 11 & 1.8 \\
\hline 2007 & 140 & 10 & 128 & 2 & 7.2 & 280 & 11 & 269 & 0 & 3.9 \\
\hline 2008 & 143 & 14 & 126 & 3 & 10.0 & 284 & 9 & 267 & 8 & 3.3 \\
\hline 2009 & 156 & 18 & 138 & 0 & 11.5 & 302 & 14 & 288 & 0 & 4.6 \\
\hline 2010 & 156 & 11 & 144 & 1 & 7.1 & 306 & 10 & 291 & 5 & 3.3 \\
\hline 2011 & 150 & 12 & 138 & 0 & 8.0 & 303 & 4 & 299 & 0 & 1.3 \\
\hline 2012 & 162 & 11 & 150 & 1 & 6.8 & 316 & 3 & 313 & 0 & 0.9 \\
\hline 2013 & 150 & 12 & 138 & 0 & 8.0 & 299 & 6 & 293 & 0 & 2.0 \\
\hline 2014 & 161 & 10 & 150 & 1 & 6.3 & 315 & 10 & 305 & 0 & 3.2 \\
\hline Overall & 2528 & 219 & 2271 & 38 & 8.8 & 6007 & 154 & 5632 & 221 & 2.6 \\
\hline
\end{tabular}

${ }^{1}$ All percentages are over the number of participants for which we were able to determine gender; in other words, we ignore participants with no gender assignment. 


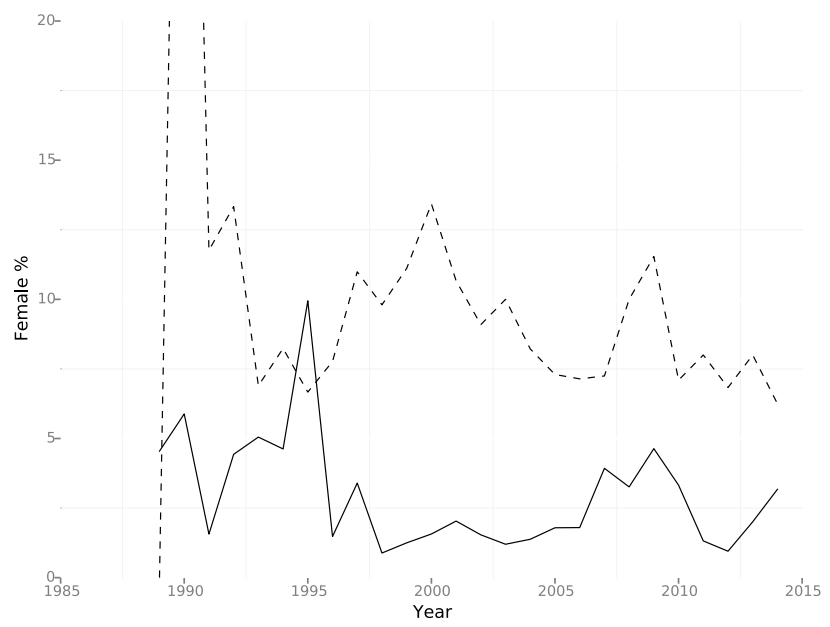

Fig. 1. Percentages of female participation by year, for contestants (solid line) and leaders (dashed line).

On the other hand, it is very visible the peak in 1995 for female contestants, caused by the rule allowing delegations with 5 contestants if the team was gender mixed. It is interesting to notice that this rule was in reaction to the low female participation in the previous years, but the situation before 1995 was much better than after (the average female participation was $4.4 \%$ in $1989-1994$ and $2.2 \%$ in 1996-2014).

Per country results. Fig. 2 is a histogram of the number of countries by percentage of female contestants ${ }^{2}$, after filtering for countries with at least 40 contestants. Among the 69 remaining countries, 22 have never had a female contestant, and only 6 countries have had more than $5 \%$ of female contestants. The country clearly differentiating, bringing to the IOI three times as many female contestants as the second ranked, are the Netherlands, those delegations are composed of females for the $22 \%$.

Performances. One of the reason that the experiment in 1995 was not repeated was that countries had difficulties in recruiting female contestants able to compete with their

Table 2

Percentages of contestants obtaining a medal, by gender

\begin{tabular}{lllll}
\hline Gender & No medal (\%) & Bronze (\%) & Silver (\%) & Gold (\%) \\
\hline Female & 77.9 & 9.1 & 11.0 & 1.9 \\
Male & 49.2 & 25.1 & 17.0 & 8.6 \\
\hline
\end{tabular}

\footnotetext{
${ }^{2}$ Here we ignore the fact that contestants and leaders can participate in multiple years, and we treat these cases as if they were different participants. Therefore, to simplify the language, we may write "a contestant" instead of "a participation of a contestant in a certain year".
} 


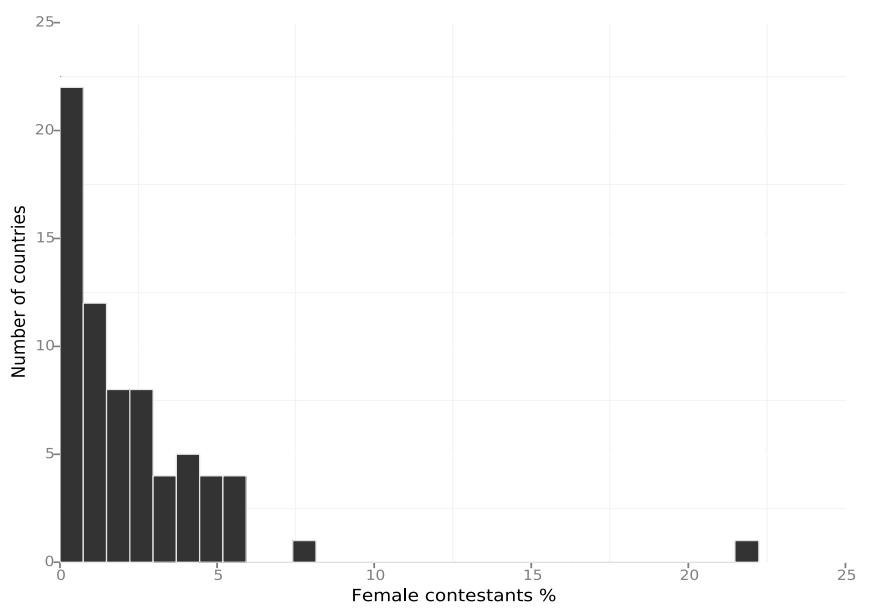

Fig. 2. Histogram of number of countries by percentages of female contestants. Only countries with at least 40 contestants are shown, which reduces the total number of countries to 69 .

male counterparts. We cannot offer statistics on the distribution of scores, because for most editions the ranking is available only for contestants with a medal. Table 2 instead shows the percentage of female and male contestants that obtained a medal. Indeed, we can see that the percentages of female contestants not receiving an award is much higher than for males. An interesting phenomenon, for which we do not have an explanation apart from the small sample, is that female contestants received more silver medals than bronze medals.

At the national level. We asked in the questionnaire about the female presence at three stages of the national selection in the last year: the "base" (at the first selection), the "national olympiad" (at the main national competition), and the "training" (when pupils receive lectures to improve their chances at the IOI).

Due to the wildly varying ways in which national selections works, it is difficult to unify the 30 responses received to this question in a single outlook. Nonetheless, some observations can still be made.

- Female participation declines as the selection progresses. This is quite expected, as the competition's base is in high school, where the gender ratio is more homogeneous than at universities and in IT companies. The last selection stage, training, shows an average female participation only slightly higher than at the IOI. At the first stage instead, many countries have double-digit female participation, and some reach parity.

- Female participation at the training level is highly correlated with programs trying to increase it. We also asked delegations to describe their actions to equalize the gender ratio, and the presence of such programs was correlated with a doubledigit presence of females at the training level. Again, this is expected, but it is nice to see that these programs have results. 
Programs to improve the gender ratio. Ten countries described programs geared towards, or having the effect of improving the ratio of female contestants. The following are the main ideas in these programs.

1. Extra training and competitions. Three countries have or had training camps and competitions aimed specifically at attracting females. This can be very helpful for promising students that might not have the occasion to try programming and computer science at all.

2. Increase of the selection pool. Two countries collaborated with math competitions for high school students to attract more talent. The gender ratio in these competitions is usually more balanced, and many of these students (regardless of gender) have potential and discover being interested in computer science despite not having any background in programming.

3. Use of non-conventional task types. The selection process can emphasize experience (that male participants are more likely to have). This can be avoided by using different type of tasks, for example mathematical, geometric, or graphical, especially at the initial levels.

\section{Comparison}

We showed that female participation at the IOI is low in absolute number: on average, $2.6 \%$ of the contestants and $8.8 \%$ of the leaders are female. Still, this could be common in the field and not specific to the IOI. The situation of women in IT and computer science, both in education and in the workforce, is indeed far from perfect, but the reality is that it is difficult to find environments as unbalanced as the IOI.

Education. In 1980, Bachelor Degrees ${ }^{3}$ in Computer Science awarded to women were about $35 \%$ of the total (Camp, 2001). This number steadily declined in the following years; in 2011, they were about $12 \%$ of the total (The Computing Research Association). Masters Degrees and Ph.D. in Computer Science have higher percentages: again in 2011 , respectively $25 \%$ and $18 \%$ were awarded to women (same source). If we include Information Sciences, percentages increase to $18 \%$ for Bachelor Degrees, $27 \%$ for Master Degrees, and 19\% for Ph.D. (National Center for Education Statistics, 2014). For a comparison, Bachelor Degrees in Mathematics awarded to women have been quite stable in the past 30 years oscillating between $40 \%$ and $47 \%$.

One might object that the IOI are an elite competition, and that might explain the difference with the percentages of Degrees awarded, but similar numbers hold for elite universities, like Stanford (Smythe, 2012).

The female presence at the International Mathematical Olympiad is steadily increasing since the 1970s, and is now just below 10\% (International Mathematical Olympiad).

\footnotetext{
3 For availability reasons, the numbers mentioned in this section will refer to the United States. Without implying that the United States are representative for the whole world, we can agree they are a leading and influential country for both computer science education and IT companies.
} 
Workforce. In academia, in 2012, women comprised $25 \%$ of all computer science assistant professors, $18 \%$ of associate professors, and $13 \%$ of full professors, and these numbers are increasing (National Center for Women and Information Technology, 2014).

Even looking at the recipients of the most prestigious awards, the female presence is higher than at the IOI: as of 2014, 3 out of 62 winners of the Turing Award were women, for a percentage of $4.8 \%$.

In the private sector, percentages are slightly higher, at around $30 \%$ for "computer and mathematics" professions. Some top IT companies (Double Union) recently divulged diversity data for their tech employees: the figures are lower, ranging between $15 \%$ and $20 \%$, but again much higher than the IOI's.

\section{Why Should We Care?}

Female participation at the IOI is much lower than in post-secondary education and in the workforce, even in top institutions and companies. This comparison should already give a warning sign, but there are also other, more significant, reasons to improve gender balance.

Promoting computer science. One of the goal of the IOI is to promote computer science among young people. Since the first editions, the number of students involved in the IOI increased exceptionally, thanks to many countries joining the competition, and to countries already participating that managed to reach more and more students.

We would claim that many countries are now hitting a wall, already reaching most students that would like to participate in the IOI. The obvious next step is to encourage students that do not know that they would like to participate; and given its current low participation rate, the group that has the most headroom for growth is that of female students.

Increasing the performance of the team. A common argument is that regardless of the effort, eventually one will need to face that girls show less interest in computer science and programming than boys.

This might be true, but not a reason not to do anything about it. For a start, the situation has not always been like this, it is enough to look at the numbers of Bachelor Degrees awarded to women in the 1980s, or at the fact that many women have been pioneers in the field of computer science (The Ada project).

Also, the correlation between national level programs and female presence at the training camps shows that it is possible to attract female contestants with the necessary skills to be part of the national team at the IOI. Indeed, one good selfish reason for establishing these programs is that increasing the pool of candidates can also increase the average level of the team, and therefore of the IOI.

Numerous researches show that gender mixed teams work better (Simard, 2007). This is admittedly a stretch for the IOI, as the competition is individual; nonetheless, training camps are social events and, in our experience, the learning approach is very 
collaborative, with a lot of horizontal knowledge distribution. Incidentally, increasing horizontal collaboration is among the steps taken by universities that have been successful in recruiting more women, like for example by implementing pair programming (National Center for Women and Information Technology, 2014).

Fairness. It is established that most people have implicit stereotypes that influence their decisions even if these prejudgments do not arise at a conscious level. For women in computing, a research showed that $70 \%$ to $80 \%$ of the subjects, regardless of gender, have implicit stereotypes associating science and tech with males more than females (Nelson, 2014).

Having unconscious biases is difficult to avoid, therefore it is important to be aware of having them and to limit their influence on our conscious decisions.

Another factor limiting fairness is stereotype threat, that is, situations that make people feel to be at risk of confirming the negative stereotypes about their group. A very relevant example is when a group is a strong minority and does not reach a critical mass.

Experiments show that performance is lower when stereotype threat is present, and higher when it is counterbalanced by a credible narrative of the path to success, by reminding of positive stereotypes, and the possibility of self-improvement, by highlighting positive examples, and role models (The Ada project).

\section{What Can We Do?}

\subsection{Principles}

Based on the previous section, we can define some guiding principles for actions that can have a positive impact on gender balance. From these we will derive the suggestions proposed for the IOI organization at national and international levels.

- Fight bias (unconscious and not). The IOI are driven by automatic scoring, that is not biased by definition. At the early stages though, there are many occasions in which bias might play a role: contests with subjective judging, decisions on which students to focus on, or which students have the most potential, etc.

- Nurture potential not already expressed. It is true that female students, like other socioeconomic groups, are in general less involved in programming courses, and are less likely to try programming on their own. Nonetheless, with the adequate motivation and training, hidden potential can develop, creating contestants able to compete for a place in the team.

- Provide a welcoming environment for everybody. Especially at the training level, teachers should take an active role in creating a welcoming environment. This does not only mean being inclusive, but also opposing stereotypes, providing positive examples, and creating a critical mass to fight isolation. 


\subsection{National Level}

Here we suggest some concrete steps that can be applied to IOI selection and training at a national level.

- Take an unconscious bias test. It is important to be aware of unconscious biases one might have, and take the necessary countermeasures. An implicit bias test is a good starting point; for example Project Implicit (Greenwald et al.) helps highlighting conscious or unconscious biases on, for example, gender and science inclination.

- Attract talented students without programming knowledge. Unconventional programming tasks, or mathheavy problems, might help identifying talented students that did not have the opportunity or motivation to practice programming.

- Collaborate with math competitions and other similar activities. Many students passionate and talented in mathematics simply have never had the opportunity or the motivation, to start practicing programming and studying computer science. This is true regardless of the gender, but math competitions tend to have a more balanced gender ratio.

- Offer entry level training for younger students. This goes hand in hand with the previous points: when the goal is to attract students without an explicit knowledge in programming, it is a necessity to give them the opportunity to learn.

- Showcase gender diversity. If not already present, invite female teachers to training camps, and create opportunities to talk about important women in the history of computer science.

\subsection{International Level}

The most significant initiative the IOI has taken was the temporary rule allowing delegations of five contestants if gender mixed ${ }^{4}$. A rule in this spirit (either by forcing gender mixed teams, or by "gifting" them with one additional contestant) was promoted by the delegation of the Netherlands since 1992; despite having support from most members of the International Committee, a consensus was not reached, and the proposal did not pass.

In 1995 the Netherlands hosted IOI and obtained funding for a fifth contestant, therefore the rule was implemented "for free" in that year. The participation to this program was positive: among the 44 delegations with at least 4 contestants, $20(45 \%)$ had a team of 5 .

On the other hand, some delegations were concerned by the difficulty in recruiting competitive female contestants. Moreover, there were loud concerns about the consequences of such a rule. In particular, that it could lead to the perception that females are

\footnotetext{
4 All the information regarding this program are extracted from reports and regulations available at the IOI website, and from personal communications with Ries Kock.
} 
less qualified than males, and to more segregation at a national level (like having separate selections for males and females). Because of this, and most probably also due to a lack of funding, the rule was not replicated in any of the following editions, remaining a single episode in the history of the IOI.

We believe that it is time to reintroduce the requirement for complete teams to be gender mixed.

On a practical level, the IOI in 1995 was very young, and it is safe to assume that the pool of students reached by the national selections was much smaller, and that high schools were much less likely to provide programming (or even computer literacy) classes. This, together with the fact that the rule was announced just one year earlier, probably made difficult for national delegations to find competitive female students.

We believe that now, with larger bases for the national selections, established training programs, and more widespread opportunities to learn to program, it would not be as hard as it was in 1995 to find suitable female students, especially if given two or three years to prepare.

The fundamental idea behind this proposal is not that we want the IOI to have a stronger female presence, and therefore we impose it with artificial rules. On the contrary, we want a higher, self-sustained, female presence because it is fair, and it is the easiest way to expand the reach of the IOI. But we feel that the process towards this goal requires a bootstrapping phase, in which countries needs to be encouraged to devise programs like those outlined before. Our hope is that this rule will lose its reason to exist in just a few years.

\section{Conclusions}

With the help of the delegations that answered our questionnaire, we showed that the female participation at the IOI is very low, even comparing it with relevant academic institutions and workplaces; but we also found evidence that programs to improve female participation actually make a difference in the number of female contestants reaching the training phase of the national selections.

We argued that increasing the participation of female students is the most natural way of continuing to fulfill the goal of the IOI of promote the discipline of informatics among young people, and eventually to improve the performances of the teams by increasing the pool of candidates. We also presented evidences that the performances of female contestants can be improved through training programs, and by removing stereotype threat (for example, creating a critical mass).

Finally, we suggested to reintroduce the requirement of mixed gender for complete teams, as a way of encouraging countries to put in place programs promoting gender balance, arguing that most countries have now a more mature selection process, able to cope with this requirement, especially if given enough time to prepare. 


\section{Acknowledgments.}

We would like to thank Ries Kock for kindly providing some background on the 1995 edition hosted by the Netherlands; Nandana Dutt and Selen Basol for useful discussions and for suggesting bibliography sources; Flavia Poma for reading the draft and suggesting several improvements; and all the people answering to the questionnaire, many of whom also communicated their support to this initiative.

\section{References}

Camp, T. (2001). Women in computer science: reversing the trend. Colorado School of Mines. http://www-2.cs.cmu.edu/ women/resources/aroundTheWeb/hostedPapers/ Syllabus-Camp.pdf

Double Union . Open diversity data. http: / / opendiversitydata .org/

Greenwald, T., Banaji, M. and Nosek, B. Project implicit. https://implicit.harvard.edu/implicit/takeatest.html

International Mathematical Olympiad. Timeline. https://www.imo-official.org/organizers.asp

Kalinicenko, E. International Olympiad in Informatics - Statistics. http://stats.ioinformatics.org

National Center for Education Statistics. (2014). Digest of Education Statistics. http://nces.ed.gov/programs/digest/2014menu_tables.asp

National Center for Women and Information Technology. (2014). NC WIT scorecard. http://www.ncwit.org/sites/default/files/resources/ncwitscorecard_081220 14 lowres.pdf

Nelson, B. (2014). The data on diversity. Communications of the ACM. http://cacm.acm.org/magazines/2014/11/179827-the-data-on-diversity/ fulltext\#R5

Simard, C. (2007 ).Barriers to the advancement of technical women. Anita Borg Institute for Women and Technology.

Smythe, S. (2012). Stanford CS Department strives for gender parity. The Stanford Daily. http://www.stanforddaily.com/2012/10/19/stanford-cs-departmentstrives-for-gender-parity/

The Ada project. Pioneering women in computing technology. http://www.women.cs.cmu.edu/ada/Resources/Women/

The Computing Research Association. Computing degree and enrollment trends. http://www.cra.org/uploads/documents/resources/taulbee/CS_Degree_and_ Enrollment_Trends_2010-11.pdf

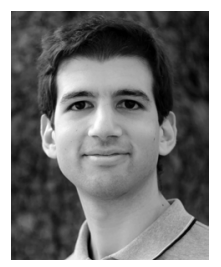

S. Maggiolo is a software engineer at Google and holds a Ph.D. in Geometry from SISSA/ISAS, Trieste. He participated in IOI 2002, winning a bronze medal and in IOI 2003. From 2006 to 2013 he collaborated with the training and selection process for the Italian team at IOI, and has been Observer in IOI 2009, Deputy Leader of the Italian team in IOI 2011 and a HSC member for IOI 2012 and IOI 2014. 